\title{
NOTES ON PROJECTIVE STRUCTURES ON COMPLEX MANIFOLDS
}

\section{BJÖRN GUSTAFSSON AND JAAK PEETRE}

\section{Introduction}

Consider a Riemann surface $X$ equipped with a projective structure, that is, a covering of $X$ with coordinate neighborhoods $U$ and corresponding (holomorphic) local coordinates $\{t\}$ such that in the intersection $U \cap U^{\prime}$ of any two such coordinate neighborhoods $U$ and $U^{\prime}$ change of local coordinates is mediated by a fractional linear transformation

$$
t^{\prime}=\frac{a t+b}{c t+d} \quad(a d-b c=1) .
$$

Because of the presence of this structure it is possible to define for each integer $\mu \geq 0$ in an invariant manner a differential operator $L_{\mu}$ which to $(-(\mu-1) / 2)$-forms ("integrals") assigns $((\mu+1) / 2)$-forms ("differentials"): If $f=f(t)(d t)^{-(\mu-1) / 2}$ then $L_{\mu} f=f^{(\mu)}(t)(d t)^{(\mu+1) / 2}{ }^{1} \quad$ The existence of the operator $L_{\mu}$ is basic for "Eichler cohomology" [E] (see also e.g. [K]) but goes back to Bol's paper [B] (cited by Eichler), so it is perhaps appropriate to speak of the "Bol operator". ${ }^{2} L_{1}$ is independent of the projective structure: If $z$ is a local coordinate, defined in some coordinate neighborhood $V$, and $f=f(z)$ then $L_{1} f \equiv d f=f^{\prime}(z) d z$. And so is $L_{0} \equiv \mathrm{id}$. Not so for $\mu>1$. For instance, if $f=f(z)(d z)^{-1 / 2}$ then $L_{2} f=\left(f^{\prime \prime}(z)+q(z) f(z)\right)(d z)^{3 / 2}$, for a suitable $q$ (depending on $V$ ). Conversely, by the knowledge of the functions $\{q(z)\}$ for a covering with coordinate neighborhoods $\{V\}$ with corresponding coordinate functions $\{z\}$ the projective structure is uniquely determined. In these notes we address ourselves, among other things, to

Received December 23, 1987.

1 To be able to define half integer forms additional information is needed, essentially the choice of a square root of the canonical sheaf $\kappa$ on $X$. More generally, if $\lambda$ is a sheaf on $X$ such that $\lambda^{2}$ differs from $\kappa$ only by a flat sheaf, one can canonically define sheaf homomorphisms $L_{\mu}: \lambda^{1-\mu} \rightarrow \lambda^{1-\mu} \otimes \kappa^{\mu}$.

${ }^{2}$ We have a short exact sequence of sheaves $0 \rightarrow \prod_{\mu-1} \rightarrow \lambda^{1-\mu} \rightarrow \lambda^{1-\mu} \otimes \kappa^{\mu} \rightarrow 0$, so the Eichler cohomology group is defined as $H^{1}\left(X, \prod_{\mu-1}\right)$. 
the problem of expressing $L_{\mu} f$ in terms of arbitrary local coordinates $z$. We show in Section 2 that if $f=f(z)(d z)^{-(\mu-1) / 2}$ then

$$
L_{\mu} f=\left(f^{(\mu)}(z)+a_{2} f^{(\mu-2)}(z)++\cdots+a_{\mu} f(z)\right)(d z)^{(\mu+1) / 2}
$$

where $a_{i}=a_{\mu}^{i}(i \geq 2)$ are certain universal polynomials in $q(z), q^{\prime}(z), \cdots$, $q^{(i-2)}(z)$. We also write abusively, suppressing $z$ (and $d z$ ),

$$
L_{\mu} f=f^{(\mu)}+a_{2} f^{(\mu-2)}+\cdots+a_{\mu} f .
$$

For instance,

$$
\begin{gathered}
L_{3} f=f^{\prime \prime \prime}+4 q f^{\prime}+2 q^{\prime} f, \\
L_{4} f=f^{I V}+10 q f^{\prime \prime}+10 q^{\prime} f^{\prime}+\left(9 q^{2}+3 q^{\prime \prime}\right) f
\end{gathered}
$$

and so forth. In Section 3 we consider briefly the special case when $X$ is a complex torus.

For completeness we begin however in Section 1 by reviewing several proof's of Bol's lemma. Section 4 is devoted to the case of several variables of this result. As an application we obtain (Section 5) a new interesting metric for holomorphic function in the unit ball of $C^{n}$. In Section 6 we consider the transformation theory of second order total p.d.e. In particular, we suggest a definition on an n-dimensional analogue of the Schwarzian.

The remainder of the paper is devoted to somewhat different, but related issues.

Section 7 begins by recalling a certain bilinear covariant due to Gordan [Go], known as the transvectant. As a byproduct we recover then the Laguerre-Forsythe invariants connected with a general $\mu$ th order linear differential operator. We obtain also (Section 8) a certain form $\Phi$ of bidegree $(0,(\mu-1) / 2)$ associated with any Hermitean metric on the Riemann surface $X$. With the aid of $\Phi$ we can prove a certain integral formula involving the Bol operator-it is a kind of "Green's formula" for $L_{\mu}$-which will do us great service in subsequent work [GB] devoted to Hankel forms on multiply connected plane domains. In Section 9 we indicate a canonical factorization of the Bol operator in the presence of a (not necessarily holomorphic) affine connectoin. Finally, Section 10 contains some simple observations about Hermitean metrics of constant curvature. 


\section{§1. Bol's lemma}

By this we intend the following result.

LEMma. If $f$ is an arbitrary (holomorphic) function and if we set

$$
g(t)=f(\phi(t))(E(t))^{\mu-1},
$$

where

$$
\phi(t)=\frac{a t+b}{c t+b}
$$

(where $a d-b c=1$ ) is a fractional linear transformation and

$$
E(t) \equiv c t+d
$$

then

$$
g^{(\mu)}(t)=f^{(\mu)}(\phi(t))(E(t))^{-(\mu+1)} .
$$

It is clear that this secures, in particular, the existence of the Bol operator on a Riemann surface equipped with a projective structure.

Below we review several proofs of this result.

In a way Bol's lemma is just a reflexion of the well known fact that the set of all polynomials of degree $<\mu$ is invariant under the group $(S L(2, C))$ action

$$
f(t) \longmapsto f(\phi(t))(E(t))^{\mu-1} .
$$

More precisely a proof can be based on Taylor's theorem. Write formally $f=T+R$ where $T$ is the Taylor polynomial of $f$ at the point $t_{0}$ and $R$ the Taylor remainder, $R(t)=O\left(\left(t-t_{0}\right)^{\mu}\right)$. By the previous observation this formula is invariant under the action (3) so that in particular $R(\phi(t))(E(t))^{\mu-1}$ must be the remainder of $f(\phi(t))(E(t))^{\mu-1}$ at the point $\phi^{-1}\left(t_{0}\right)$. This readily gives the desired conclusion.

The simplest proof goes perhaps as follows. It suffices to take

$$
f(t)=\frac{1}{u-t}
$$

Then

$$
g(t)=\frac{1}{v-t} E(v)(E(t))^{\mu}
$$

if $u=\phi(v)$, as 


$$
\phi(v)-\phi(t)=\frac{v-t}{(c t+d)(c v+d)}=\frac{v-t}{E(t) E(v)} .
$$

Also

$$
E(t)=E(v)+c(t-v) .
$$

That is,

$$
\begin{aligned}
g(t) & =\frac{(E(v))^{\mu+1}}{v-t}+\text { polynomial of degree }<\mu, \\
g^{(\mu)}(t) & =\frac{\mu !(E(v))^{\mu+1}}{(v-t)^{\mu+1}} .
\end{aligned}
$$

On the other hand

$$
f^{(\mu)}(t)=\frac{\mu !}{(u-t)^{\mu+1}}, \quad\left(f^{(\mu)} \circ \phi\right)(t)=\frac{\mu !}{(v-t)^{\mu+1}}(E(v))^{\mu+1}(E(t))^{\mu+1} .
$$

Then (2) follows.

Alternative proof. Use more directly Cauchy's formula

$$
f(t)=\frac{1}{2 \pi i} \int \frac{f(u)}{u-t} d u
$$

One can also connect Bol's theorem with the invariance properties of Newton's divided differences. Let us put

$$
\begin{aligned}
(F(t) & =f(t),) \\
F\left(t_{1}, t_{2}\right) & =\frac{f\left(t_{1}\right)-f\left(t_{2}\right)}{t_{1}-t_{2}}, \\
F\left(t_{1}, t_{2}, t_{3}\right) & =\frac{F\left(t_{1}, t_{2}\right)-F\left(t_{1}, t_{3}\right)}{t_{2}-t_{3}},
\end{aligned}
$$

It is well-known and easy to prove (see e.g. [N]) that

$$
F\left(t_{1}, t_{2}, \cdots, t_{\mu+1}\right)=\frac{\mu !}{2 \pi i} \int \frac{f(u)}{\left(u-t_{1}\right) \cdots\left(u-t_{\mu+1}\right)} d u,
$$

integrating over a suitable cycle. This shows in particular that

$$
F(t, t, \cdots, t)=f^{(\mu)}(t) .
$$

As before we show that if $g$ is given by (1) and $G$ corresponds to $g$ (in the same way as $F$ corresponds to $f$ ) then 


$$
G\left(t_{1}, \cdots, t_{\mu+1}\right)=F\left(\phi\left(t_{1}\right), \cdots, \phi\left(t_{\mu+1}\right)\right)\left(E\left(t_{1}\right)\right)^{-1} \cdots\left(E\left(t_{\mu+1}\right)\right)^{-1} .
$$

In the limit we obtain (2).

Bol's theorem can also be obtained as a special case $(k=\mu)$ of the following more general result (see e.g. $[\mathrm{M}],[\mathrm{T}]$ ):

LEMMA. We have

$$
g^{(k)}(t)=\sum_{j=0}^{k} \alpha_{\jmath}^{k} f^{(k-j)}(\phi(t))(E(t))^{\mu-1-2 k+j} c^{j}
$$

where

$$
\alpha_{j}^{k}=\left(\begin{array}{c}
k \\
j
\end{array}\right)(\mu-k)(\mu-k+1) \cdots(\mu-k+j-1) .
$$

First proof. By induction we realize that there must exist a recursion formula of the type (5) with coefficients $\alpha_{j}^{k}$ given by

$$
\alpha_{j}^{k+1}=\alpha_{j}^{k}+(\mu-1-2 k+j-1) \alpha_{j-1}^{k}
$$

and initial condition.

$$
\alpha_{0}^{k}=1 \text {. }
$$

Another induction shows that these are precisely given by (6).

Second proof. Write

$$
g(t+s)=f(\phi(t+s))(E(t+s))^{\mu-1}
$$

and consider the Taylor development of both sides taking into account that

$$
\phi(t+s)=\phi(t)+\frac{s}{E(t) E(t+s)}
$$

and

$$
E(t+s)=E(t)+c s .
$$

Indeed, the coefficient of $s^{k}$ to the left is

$$
\frac{1}{k !} g^{(k)}(t)
$$

and to the right

$$
\sum_{j=0}^{k} \frac{1}{(k-j) !} f^{(k-j)}(\phi(t))\left(\begin{array}{c}
\mu-1-k+j \\
j
\end{array}\right)(E(t))^{\mu-1-2 k+j} c^{j}
$$


Equating these two expressions the desired expression for $\alpha_{j}^{k}$ readily follows.

A third proof would imitate the proof of Bol's lemma we originally gave but this becomes somewhat cumbersome. Instead we reverse the procedure and use the lemma to derive a combinatorial indentity.

Take again

$$
f(t)=\frac{1}{u-t}
$$

so that

$$
g(t)=\frac{E(v)(E(t))^{\mu}}{v-t}
$$

if $u=\phi(v)$. Then

$$
f^{(k-j)}(t)=\frac{(k-j) !}{(u-t)^{k-j+1}}
$$

and

$$
\left(f^{(k-j)} \circ \phi\right)(t)=\frac{(k-j) !}{(v-t)^{k-j+1}}(E(v))^{k-j+1}(E(t))^{k-j+1}
$$

so that the right hand side of (5) is

$$
\sum_{j=0}^{k} \alpha_{j}^{k} c^{j} \frac{(k-j) !}{(v-t)^{k-j+1}}(E(v))^{k-j+1}(E(t))^{\mu-k} .
$$

As

$$
(E(t))^{\mu-k}=(E(v)+c(t-v))^{\mu-k}=\sum_{h=0}^{\mu-k}\left(\begin{array}{c}
\mu-k \\
h
\end{array}\right) c^{h}(t-v)^{h}(E(v))^{\mu-k-h},
$$

we may write this as

$$
\sum_{j=0}^{k} \alpha_{j}^{k}(k-j) ! \sum_{h=0}^{\mu-k}(-1)^{h}\left(\begin{array}{c}
\mu-k \\
h
\end{array}\right) c^{j+h} \frac{(E(v))^{\mu-j-h+1}}{(v-t)^{k-j-h+1}}
$$

or, again setting $j+h=l$,

$$
\sum_{l=0}^{\mu} \frac{c^{l}(E(c))^{\mu-l+1}}{(v-t)^{k-l+1}} \sum_{j=l-(\mu-k)}^{l}(-1)^{l-j}(k-j) !\left(\begin{array}{c}
\mu-k \\
l-j
\end{array}\right) \alpha_{j}^{k} .
$$

On the other hand, writing 


$$
\begin{aligned}
g(t) & =\frac{E(v)(E(t))^{\mu}}{v-t}=\frac{E(v)(E(v)+c(t-v))^{\mu}}{v-t} \\
& =\frac{E(v)}{v-t} \sum_{l=0}^{\mu}\left(\begin{array}{l}
\mu \\
l
\end{array}\right) c^{l}(t-v)^{l}(E(v))^{\mu-l} \\
& =\sum_{l=0}^{\mu}(-1)^{l}\left(\begin{array}{l}
\mu \\
l
\end{array}\right) c^{l}(v-t)^{l-1}(E(v))^{\mu-l+1},
\end{aligned}
$$

we find

$$
g^{(k)}(t)=\frac{k !(E(v))^{\mu+1}}{(v-t)^{k+1}}+\sum_{l=k+1}^{\mu}(-1)^{l-k}\left(\begin{array}{c}
\mu \\
l
\end{array}\right) \frac{(l-1) !}{(l-k-1) !} \frac{c^{l}(E(v))^{\mu-l+1}}{(v-t)^{k-l+1}} .
$$

Comparison of (7) and (8) gives

$$
\begin{aligned}
& \sum_{j=l+k-\mu}^{\mu}(-1)^{l-j}(k-j) !\left(\begin{array}{c}
\mu-k \\
l-j
\end{array}\right) \alpha_{j}^{k} \\
& = \begin{cases}k ! & \text { for } l=0 \\
0 & \text { for } 0<l \leq k \\
(-1)^{l-k}\left(\begin{array}{l}
\mu \\
l
\end{array}\right) \frac{(l-1) !}{(l-k-1) !} & \text { for } k+1 \leq l \leq \mu .\end{cases}
\end{aligned}
$$

These equations for $0 \leq l<k$ determine also the $\alpha_{j}^{k}$ uniquely.

\section{$\S 2$. The Bol operator in general coordinates}

Let now $z=\phi(t)$ be an arbitrary invertible function (change of variable). It is convenient to put

$$
E(t)=\frac{1}{\sqrt{\phi^{\prime}(t)}}
$$

If we put

$$
g(t)=f(\phi(t))(E(t))^{\mu-1}
$$

then a differential operator $L_{\mu}$ is defined via the formula

$$
g^{(\mu)}(t)=L_{\mu} f(\phi(t))(E(t))^{-(\mu+1)} .
$$

Our concern is to find an explicit formula for $L_{\mu}$. To this end we introduce the quantities

$$
r=E \cdot D E \text { and } q=E^{3} \cdot D^{2} E(D=d / d t),
$$

which are regarded as functions of $z$. Let us notice that 


$$
\frac{d r}{d t}=D r=(D E)^{2}+E D^{2} E=E^{-2}\left((E \cdot D E)^{2}+E^{3} L^{2} E\right)=E^{-2}\left(r^{2}+q\right),
$$

that is, we have the Riccati equation

$$
\frac{d r}{d z}=r^{2}+q
$$

Take now two times derivatives in (0)!

$$
\begin{aligned}
g^{\prime} & =f^{\prime} E^{\mu-3}+f(\mu-1) E^{\mu-2} D E \\
& =\left(f^{\prime}+(\mu-1) E D E f\right) E^{\mu-3} \\
& =\left[f^{\prime}+(\mu-1) r f\right] E^{\mu-3},
\end{aligned}
$$

$$
\begin{aligned}
g^{\prime \prime} & =[]^{\prime} E^{\mu-5}+[](\mu-3) E^{\mu-4} D E \\
& =\left[f^{\prime \prime}+(\mu-1) r f^{\prime}+(\mu-1) r^{\prime} f+(\mu-3) r f^{\prime}+(\mu-1)(\mu-3) r^{2} f\right] E^{\mu-5} \\
& =\left[f^{\prime \prime}+2(\mu-2) r f^{\prime}+\left((\mu-1)(\mu-2) r^{2}+(\mu-1) q\right) f\right] E^{\mu-5} .
\end{aligned}
$$

Putting $\mu=0,1,2$ in (0), (1), (2) we find

$$
L_{0} f=f, \quad L_{1} f=f^{\prime}, \quad L_{2} f=f^{\prime \prime}+q f .
$$

Remark. This shows that the Bol operator on a Riemann surface corresponding to a projective structure on that surface is independent of the latter if $\mu=0,1$ and determines it if $\mu=2$. (Given a second order operator locally of the form $L f=f^{\prime \prime}+q f$ then there exist new local coordinates such that it in these coordinates takes the form $L f=f^{\prime \prime}$. See further the discussion in Section 9.)

Continue the derivation!

$$
\begin{aligned}
g^{\prime \prime \prime}= & {[]^{\prime} E^{\mu-7}+[](\mu-5) E^{\mu-6} D E } \\
= & {\left[f^{\prime \prime \prime}+2(\mu-2) r f^{\prime \prime}+2(\mu-2) r^{\prime} f^{\prime}+\left((\mu-1)(\mu-2) r^{2}+(\mu-1) q\right) f^{\prime}\right.} \\
& +\left((\mu-1)(\mu-2) 2 r r^{\prime}+(\mu-1) q^{\prime}\right) f+(\mu-5) r f^{\prime \prime} \\
& +2(\mu-2)(\mu-5) r^{2} f^{\prime}+(\mu-1)(\mu-2)(\mu-5) r^{3} f \\
& +(\mu-1)(\mu-5) q r f] E^{\mu-7} \\
= & {\left[f^{\prime \prime \prime}+3(\mu-3) r f^{\prime \prime}+\left(3(\mu-2)(\mu-3) r^{2}+(3 \mu-5) q\right) f^{\prime}\right.} \\
& \left.+\left((\mu-1)(\mu-2)(\mu-3) r^{3}+3(\mu-1)(\mu-3) r q+(\mu-1) q^{\prime}\right) f\right] E^{\mu-7} .
\end{aligned}
$$

Putting $\mu=3$ gives

$$
L_{3} f=f^{\prime \prime \prime}+4 q f^{\prime}+2 q^{\prime} f .
$$

In the same way we find, for instance, 


$$
L_{4} f=f^{I V}+10 q f^{\prime \prime}+10 q^{\prime} f^{\prime}+\left(9 q^{2}+3 q^{\prime \prime}\right) f .
$$

In the general case it is easy to see that

$$
g^{(k)}=\left[f^{(k)}+A_{1}^{k} f^{(k-1)}+\cdots+A_{k}^{k}\right] E^{\mu-1-2 k},
$$

where the coefficients $A_{j}^{k}(j \geq 1)$ are polynomials in $\mu, r, q, q^{\prime}, q^{\prime \prime}, \cdots, q^{(j-2)}$ determined by the recursion

$$
A_{j}^{k+1}=A_{j}^{k}+[D+(\mu-1-2 k) r] A_{j-1}^{k} \quad(D=d / d z)
$$

(and the initial condition $A_{0}^{k} \equiv 1$ ). Bol's lemma (Section 1) suggests that if $k=\mu$ then all terms vanish except those which do not contain any power of $r$. In other words:

THEOREM. We have

$$
L_{\mu} f=f^{(\mu)}+a_{2}^{\mu} f^{(\mu-2)}+\cdots+a_{\mu}^{\mu},
$$

where $a_{j}^{\mu}(j \geq 2)$ is a polynomial in $q, q^{\prime}, \cdots, q^{(j-2)}$ :

$$
a_{j}^{\mu}=a_{j}^{\mu}\left(q, q^{\prime}, \cdots, q^{(j-2)}\right) .
$$

Moreover, in $a_{j}^{\mu}$ there appear only terms $q^{n_{0}}\left(q^{\prime}\right)^{n_{1}}\left(q^{\prime \prime}\right)^{n_{2}} \cdots$ with $\sum_{i}(i+2) n_{i}$ $=j$.

Proof. For the proof consider an arbitrary Möbius transformation

$$
t=\zeta(s)=\frac{a s+b}{c s+d}
$$

(with $a d-b c=1$ ) and consider the composed change of local coordinates $z=\psi(s)=\phi(\zeta(s))$. Denote by $\tilde{r}, \tilde{q}$ the quantities corresponding to $r, q$ but formed with $\psi$ instead of $\phi$. It is clear that

$$
\tilde{q}=q
$$

and a simple calculation reveals that

$$
\tilde{r}=r+\frac{c}{c s+d} \frac{1}{\psi^{\prime}(s)} .
$$

In particular $\tilde{r} \neq r$ as soon as $c \neq 0$. It is also clear that

$$
A_{k}^{\mu}\left(\tilde{q}, \tilde{q}^{\prime}, \cdots, \tilde{q}^{(k-2)}, \tilde{r}\right)=A_{k}^{\mu}\left(q, q^{\prime}, \cdots, q^{(k-2)}, r\right) .
$$

Upon varying $\zeta$ we thus see that $A_{k}^{\mu}$ is independent of $r$. 
Remark. $q$ is nothing but the Schwarz derivative in disguise. More precisely, one has in more conventional notation (see e.g. [Gu1, 2])

$$
\begin{gathered}
\{z, t\}_{1}=-2 r \frac{d z}{d t} \\
\{z, t\}=\{z, t\}_{2}=-2 q\left(\frac{d z}{d t}\right)^{2} \quad \text { (Schwarz derivative) } .
\end{gathered}
$$

One has (see again $[\mathrm{Gu1}, 2]$ )

$$
\{z, t\}_{k}(d t)^{k}=\{z, s\}_{k}(d s)^{k}+\{s, t\}_{k}(d t)^{k} \quad(k=1,2)
$$

where now $t=\zeta(s)$ need not be Möbius. In particular

$$
\{z, t\}_{k}(d t)^{k}=-\{t, z\}_{k}(d z)^{k} \quad(k=1,2)
$$

so that, alternatively, we may define

$$
r=\frac{1}{2}\{t, z\}_{1}, \quad q=\frac{1}{2}\{t, z\}_{2} .
$$

It is likewise convenient to put

$$
\{z, t\}_{0}=\log \frac{d z}{d t} .
$$

Then $(*)$ is valid also for $k=0$.

Here are some alternative expressions for these brackets if $k=1,2$ :

$$
\begin{aligned}
& \{z, t\}_{1}=-2 \sqrt{\phi^{\prime}(t)} \frac{d}{d t} \frac{1}{\sqrt{\phi^{\prime}(t)}}=\frac{d}{d t} \log \left(\frac{d z}{d t}\right) \\
& \{z, t\}_{2}=-2 \sqrt{\phi^{\prime}(t)} \frac{d^{2}}{d t^{2}} \frac{1}{\sqrt{\phi^{\prime}(t)}}=\frac{d^{2}}{d t^{2}} \log \frac{d z}{d t}-\frac{1}{2}\left(\frac{d}{d z} \log \left(\frac{d z}{d t}\right)\right)^{2} .
\end{aligned}
$$

\section{§3. An example}

Let $X$ be a compact Riemann surface of genus 1, in other words, an elliptic curve, presented as a complex torus $C / \Lambda$. The operator $L_{2}$ corresponding to a projective structure on $X$ must be of the form $L_{2}=$ $d^{2} / d z^{2}+q$ where now $q$ is a constant (a complex number). A basis for solutions to the corresponding homogeneous linear partial differential equation is given by the functions $e^{\sqrt{-q} z}, e^{-\sqrt{-q} z}$. Thus the corresponding "geometric realization" (in the sense of [Gu1,2]) is given by $t=e^{2 \sqrt{-q} z}$. The space of the kernel of the operator $L_{\mu}=d^{\mu} / d t^{\mu}$ is spanned by 


$$
\left\{1, t, \cdots, t^{\mu-1}\right\} \times(d t)^{-(\mu-1) / 2},
$$

that is, by

$$
\left\{e^{((\mu-1) / 2) 2 \sqrt{-q} z}, \cdots, e^{-((\mu-1) / 2) 2 \sqrt{-q} z}\right\} \times(d z)^{-(\mu-1) / 2} .
$$

It follows that

$$
\begin{aligned}
L_{\mu} & =\prod_{k=0}^{\mu-1}\left(\frac{d}{d z}-(\mu-1-2 k) \sqrt{-q}\right) \\
& =\left(\frac{d}{d z}\right)^{\mu}+C_{2}^{\mu} q\left(\frac{d}{d z}\right)^{\mu-2}+C_{4}^{\mu} q^{2}\left(\frac{d}{d z}\right)^{\mu-4}+\cdots \quad \text { (only "even" terms) } .
\end{aligned}
$$

This again entails that also in the general case the "leading" term in the polynomial $a_{j}^{\mu}=a_{j}^{\mu}\left(q, \cdots, q^{(j-2)}\right)$ is

$$
\begin{cases}C_{j}^{\mu} q^{j / 2} & \text { if } j \text { even }, \\ 0 & \text { if } j \text { odd } .\end{cases}
$$

Remark. Here one could also have used the general form of the coefficients in $a_{j}^{\prime \prime}$ (see the last sentence of the theorem in Section 2).

Returning to the present case ( $X$ a complex torus) we write down the operator $L_{\mu}$ in the first few cases ( $q$ constant, $D=d / d z$ )

$$
\begin{aligned}
L_{2} & =(D-\sqrt{-q})(D+\sqrt{-q})=D^{2}+q, \\
L_{3} & =D(D-2 \sqrt{-q})(D+2 \sqrt{-q})=D^{3}+4 q D, \\
L_{4} & =(D+3 \sqrt{-q})(D+\sqrt{-q})(D-\sqrt{-q})(D-3 \sqrt{-q}) \\
& =D^{4}+10 q D^{2}+9 q^{2},
\end{aligned}
$$

For a generalization of the above factorizations see Section 9 .

\section{§4. Several variables}

Consider a fractional linear transformation

$$
\phi(t)=\frac{F(t)}{E(t)}
$$

in $C^{n}$ with points $t=\left(t_{1}, \cdots, t_{n}\right) ; E(t)$ and $F(t)$ are thus "inhomogeneous" linear functions, the former scalar and the latter vector $\left(\boldsymbol{C}^{n}\right)$ valued. ${ }^{3} \mathrm{We}$ notice that

${ }^{3}$ Let $\left(\begin{array}{l}E \\ F\end{array}\right)$ denote the corresponding linear transformation in $C^{n+1}$. Then $E, F$ can be nomalized by the requirement $\operatorname{det}\left(\begin{array}{l}E \\ F\end{array}\right)=1$. 


$$
E(t+s)=E(t)+E^{\prime}(t ; s)
$$

and similarly for $F$ (in an obvious notation for first order derivatives). This gives

$$
\phi(t+s)=\phi(t)+\frac{L(t ; s)}{E(t) E(t+s)}
$$

with $L(t ; s) \equiv F^{\prime}(t ; s) E(t)-F(t) E^{\prime}(t ; s)$, a linear function in $s$. In particular, the derivative of $\phi$ is given by

$$
\phi^{\prime}(t ; s)=\frac{L(t ; s)}{(E(t))^{2}} .
$$

Remark. Notice the formula

$$
\phi^{(m)}(t ; s)=(-1)^{m-1} m ! \frac{L(t ; s)\left(E^{\prime}(t ; s)\right)^{m-1}}{(E(t))^{m+1}}
$$

for the $m$ th order derivative of $\phi$.

If $f$ is given (holomorphic) function, we define the function $g(t)$ as before by

$$
g(t)=f(\phi(t))(E(t))^{\mu-1} .
$$

Then the formula for the $k$ th derivative ((5), (6) in Section 1) generalizes as follows

$$
\begin{aligned}
g^{(k)}(t ; s) & =\sum_{j=0}^{k} \alpha_{j}^{k} f^{(k-j)}(\phi(t) ; L(t ; s))(E(t))^{\mu-1-2 k+j}\left(E^{\prime}(t ; s)\right)^{j} \\
& =\sum_{j=0}^{k} \alpha_{j}^{k} f^{(k-j)}\left(\phi(t) ; \phi^{\prime}(t ; s)\right)(E(t))^{\mu-1-j}\left(E^{\prime}(t ; s)\right)^{j}
\end{aligned}
$$

In particular $(k=\mu)$ this gives a generalization of Bol's lemma:

$$
g^{(\mu)}(t ; s)=f^{(\mu)}(\phi(t) ; L(t ; s))(E(t))^{-\mu-1}=f^{(\mu)}\left(\phi(t) ; \phi^{\prime}(t ; s)\right)(E(t))^{\mu-1} .
$$

Projective formulation. Introduce homogeneous coordinates $Z=$ $\left(Z_{0}, \cdots, Z_{n}\right)$ with $z_{i}=Z_{i} / Z_{0}(i=1, \cdots, n)$. Putting

$$
P(Z)=Z_{0}^{\mu-1} f\left(Z_{1} / Z_{0}, \cdots, Z_{n} / Z_{0}\right)
$$

we get a homogeneous function of degree $\mu-1$ on $C^{n+1}$. Its $\mu$ th derivative at the point $Z$ is a $\mu$-linear form $P^{(\mu)}(Z, \cdot)$ on $C^{n+1}$. Then our generalization of Bol's lemma entails that $P^{(\mu)}(Z, \cdot)$ descends to a $\mu$-linear form on the tangent space to $P^{n}$ at the image point $z$ of $Z$ under the 
canonical map $\boldsymbol{C}^{n+1} \backslash\{0\} \rightarrow \boldsymbol{P}^{n}$. (One has essentially to repeat the previous reasoning with $Z_{0}$ playing the rôle of $E$ and $\left\{Z_{1}, \cdots, Z_{n}\right\}$ the rôle of $F$.)

\section{Explicitly:}

$$
\begin{aligned}
P^{(\mu)}(Z, \cdot) & =d^{\mu} P(Z, \cdot) \\
& =Z_{0}^{\mu-1} \sum_{\alpha} \frac{\partial^{\mu} f\left(Z_{1} / Z_{0}, \cdots, Z_{\mu} / Z_{0}\right)}{\partial z_{\alpha_{1}} \cdots \partial z_{\alpha_{\mu}}} d\left(Z_{\alpha_{1}} / Z_{0}\right) \cdots d\left(Z_{\alpha_{\mu}} / Z_{0}\right) .
\end{aligned}
$$

As

$$
d\left(\frac{Z_{i}}{Z_{0}}\right)=\frac{d Z_{i}}{Z_{0}}-\frac{Z_{i} d Z_{0}}{Z_{0}^{2}}
$$

this gives (for $Z_{0}=1, Z_{i}=z_{i}$ ) in obvious multi-index notation:

$$
\frac{\partial^{\mu} P\left(1, z_{1}, \cdots, z_{n}\right)}{\partial Z_{0}^{j} \partial Z_{\gamma_{1}} \cdots \partial Z_{\gamma_{k}}}=(-1)^{j} \sum_{\alpha=\beta \cup_{\gamma}} z_{\beta_{1}} \cdots z_{\beta_{j}} \frac{\partial^{\mu} f\left(z_{1}, \cdots, z_{n}\right)}{\partial z_{\alpha_{1}} \cdots \partial z_{\alpha_{\mu}}} .
$$

ExAmple. Consider, for instance, the case $\mu=2$ so that

$$
P(Z)=Z_{0} f\left(Z_{1} / Z_{0}, \cdots, Z_{n} / Z_{0}\right) \text {. }
$$

Then, explicitly,

$$
\begin{gathered}
\frac{\partial P}{\partial Z_{i}}=\frac{\partial f}{\partial z_{i}} ; \quad \frac{\partial P}{\partial Z_{0}}=f-\sum_{k} \frac{Z_{k}}{Z_{0}} \frac{\partial f}{\partial z_{k}}=f-\sum_{k} z_{k} \frac{\partial f}{\partial z_{k}} ; \\
\frac{\partial^{2} P}{\partial Z_{i} \partial Z_{j}}=\frac{1}{Z_{0}} \frac{\partial^{2} f}{\partial z_{i} \partial z_{j}} ; \quad \frac{\partial^{2} P}{\partial Z_{0} \partial Z_{i}}=-\sum_{k} \frac{Z_{k}}{Z_{0}^{2}} \frac{\partial^{2} f}{\partial z_{i} \partial z_{k}}=-\frac{1}{Z_{0}} \sum_{k} z_{k} \frac{\partial^{2} f}{\partial z_{i} \partial z_{k}} ; \\
\frac{\partial^{2} P}{\partial Z_{0}^{2}}=-\sum_{k} \frac{Z_{k}}{Z_{0}^{2}} \frac{\partial f}{\partial \boldsymbol{z}_{k}}+\sum_{k} \frac{Z_{k}}{Z_{0}^{2}} \frac{\partial f}{\partial \boldsymbol{z}_{k}}+\sum_{k, l} \frac{Z_{k} Z_{l}}{Z_{0}^{3}} \frac{\partial^{2} f}{\partial \boldsymbol{z}_{k} \partial z_{l}} \\
=\sum_{k, l} \frac{Z_{k} Z_{l}}{Z_{0}^{3}} \frac{\partial^{2} f}{\partial \boldsymbol{z}_{k} \partial z_{l}}=\frac{1}{Z_{0}} \sum_{k, l} z_{k} z_{l} \frac{\partial^{2} f}{\partial \boldsymbol{z}_{k} \partial \boldsymbol{z}_{l}} .
\end{gathered}
$$

In other words:

$$
\sum_{i, j=0}^{n} \frac{\partial^{2} P}{\partial Z_{i} \partial Z_{j}} a_{i} b_{j}=\frac{1}{Z_{0}} \sum_{i, j=1}^{n}\left(a_{i}-a_{0} z_{i}\right)\left(b_{j}-b_{0} z_{j}\right) \frac{\partial^{2} f}{\partial z_{i} \partial z_{j}} .
$$

Global consequence. Let $X$ be an $n$-dimensional complex manifold equipped with a projective structure and $\lambda$ a sheaf on $X$ such that $\lambda^{n+1}=$ $\operatorname{det} \Lambda$ ( $=\kappa$, the canonical bundle of $X$ ) or, more generally, such that $\lambda^{n+1}$ differs from $\operatorname{det} \Lambda$ by a flat sheaf; $\Lambda$ is the cotangent sheaf of $X$. Then we have an invariantly defined operator $L_{\mu}: \lambda^{1-\mu} \rightarrow \lambda^{1-\mu} \otimes \Lambda^{\mu}$. This again gives an exact sequence: 


$$
0 \longrightarrow \prod_{\mu-1} \longrightarrow \lambda^{1-\mu} \longrightarrow \lambda^{1-\mu} \otimes A^{\mu} .
$$

Does there exists a natural continuation to a complex of sheaves?

\section{§5. Invariant metrics}

We can use the previous considerations (Section 4) to write down some new (?) interesting (??) metrics. Let $B$ be the (complex) unit ball in $C^{n}$, i.e. $B$ is the image of the Hermitean cone

$$
\|\boldsymbol{Z}\|^{2} \equiv Z_{0} \bar{Z}_{0}-Z_{1} \bar{Z}_{1}-\cdots-Z_{n} \bar{Z}_{n} \geq 0
$$

under the canonical projection $\boldsymbol{C}^{n+1} \backslash\{0\} \rightarrow \boldsymbol{P}^{n}$. If $\boldsymbol{P}$ is a homogeneous function of degree $\mu-1$ in $C^{n+1}$ over $B$ we set

$$
\|P\|^{2}=\int_{B}\left(\|Z\| \cdot\left\|P^{(\mu)}(Z, \cdot)\right\|\right)^{2} d I(Z),
$$

where $\left\|P^{(\mu)}\right\|$ is measured in the Hermitean metric induced by the metric in (1) and $I$ is the $S U(1, n)$ invariant (Poincaré) measure on $B(d I(z)=$ $\left.d E(z) /\left(1-\|z\|^{2}\right)^{n+1}\right)$. Explicitly (in terms of $f$ ):

$$
\begin{aligned}
\|f\|^{2}= & \int_{B}\left(1-\|z\|^{2}\right) \sum_{j=0}^{\mu}(-1)^{j}\left(\begin{array}{c}
\mu \\
j
\end{array}\right) \sum_{r}\left|\sum_{\beta} z_{\beta_{1}} \cdots z_{\beta_{j}} \frac{\partial^{\mu} f\left(z_{1}, \cdots, z_{n}\right)}{\partial z_{\alpha_{1}} \cdots \partial z_{\alpha_{\mu}}}\right|^{2} \\
& \times \frac{d E(z)}{\left(1-\|z\|^{2}\right)^{n+1}} .
\end{aligned}
$$

EXAMPles 1. $\mu=1, n$ general.

$$
\|f\|^{2}=\int_{B}\left(1-\|z\|^{2}\right)\left(\sum_{i=1}^{n}\left|\frac{\partial f}{\partial z_{i}}\right|^{2}-\left|\sum_{i=1}^{n} z_{i} \frac{\partial f}{\partial z_{i}}\right|^{2}\right) \frac{d E(z)}{\left(1-\|z\|^{2}\right)^{n+1}},
$$

that is, the $S U(1, n)$ invariant Dirichlet integral corresponding to the Bergman metric on $B$ (see e.g. [R]).

2. $\mu$ general, $n=1, B=\Delta=$ unit disk in $C$.

$$
\|f\|^{2}=\int_{\Delta}\left|\left(1-|z|^{2}\right)^{(\mu+1) / 2} \frac{d^{\mu} f}{d z^{\mu}}\right|^{2} \frac{d E(z)}{\left(1-|z|^{2}\right)^{2}} ;
$$

this case appears e.g. in [P].

\section{§6. A p.d.e. approach to uniformization}

It is a fact that uniformization in the classical case of one complex variable is intimately connected with the second order linear differential 
equation $f^{\prime \prime}+a f^{\prime}+b f=0$. This suggests to try, in the case of several variables, with total second order partial differential equations:

$$
\frac{\partial^{2} f}{\partial z_{k} \partial z_{l}}+A_{k l}^{r} \frac{\partial f}{\partial z_{r}}+B_{k l} f=0 \quad(k, l=1, \cdots, n) .
$$

(Here and in the sequel we use, whenever convenient, the Einstein summation convention.) This may be viewed as a pedestrian version of more sophisticated approaches (cf. e.g. [Gu3]).

Let $z=\phi(t)$ be a locally invertible map (change of local coordinates) and $m$ a nonvanishing function (multiplier). Put

$$
g(t)=f(\phi(t)) m(t) .
$$

The first and the second derivatives undergo the following transformation:

$$
\begin{gathered}
\frac{\partial g(t)}{\partial t_{i}}=\frac{\partial f(\phi(t))}{\partial z_{k}} \frac{\partial \phi_{k}}{\partial t_{i}} m(t)+f(\phi(t)) \frac{\partial m}{\partial t_{i}} . \\
\frac{\partial^{2} g(t)}{\partial t_{i} \partial t_{j}}=\frac{\partial^{2} f(\phi(t))}{\partial z_{k} \partial z_{l}} \frac{\partial \phi_{k}}{\partial t_{i}} \frac{\partial \phi_{l}}{\partial t_{j}} m(t) \\
+\frac{\partial f(\phi(t))}{\partial z_{k}}\left(\frac{\partial^{2} \phi_{k}}{\partial t_{i} \partial t_{j}} m+\frac{\partial \phi_{k}}{\partial t_{i}} \frac{\partial m}{\partial t_{j}}+\frac{\partial \phi_{k}}{\partial t_{j}} \frac{\partial m}{\partial t_{i}}\right)+f(\phi(t)) \frac{\partial^{2} m}{\partial t_{i} \partial t_{j}} .
\end{gathered}
$$

If we want the first derivatives to drop out in the expression for the transformed differential equation, we are led to impose the requirement

$$
\frac{\partial m}{\partial t_{j}} \frac{\partial \phi_{k}}{\partial t_{i}}+\frac{\partial m}{\partial t_{i}} \frac{\partial \phi_{k}}{\partial t_{j}}+\frac{\partial^{2} \phi_{k}}{\partial t_{i} \partial t_{j}} m=0
$$

or in more compact notation

$$
m d^{2} \phi+d m \otimes d \phi+d \phi \otimes d m=0 .
$$

We claim that this entails

$$
m=\text { const } \cdot J^{-1 /(n+1)},
$$

where

$$
J \stackrel{\text { def }}{=} \operatorname{det}\left(\frac{\partial \phi_{k}}{\partial t_{i}}\right)=\operatorname{det} \phi^{\prime} .
$$

Proof. Notice first that

$$
\frac{\partial J}{\partial t_{i}}=\sum_{j, k} \frac{\partial^{2} \phi_{k}}{\partial t_{i} \partial t_{j}}\left(\phi^{\prime}\right)_{j k}^{c o}
$$


So multiplying (3) with $\left(\phi^{\prime}\right)^{c o}$ and summing (over $j, k$ ) we obtain

$$
\frac{\partial m}{\partial t_{i}} J+\frac{\partial m}{\partial t_{i}} n J+m \frac{\partial J}{\partial t_{i}}=0
$$

which obviously yields (5).

So if the desideratum $A_{k l}^{r}=0$ can be realized we can, as in the one dimensional case, henceforth restrict ourselves to the case $m=$ const. $J^{-1 /(n+1)}$.

Return to the case of general $A, B$. From (2) and (3) follows that

$$
\begin{aligned}
\frac{\partial^{2} g}{\partial t_{i} \partial t_{j}} & +\tilde{A}_{i j}^{k} \frac{\partial g}{\partial t_{k}}+\tilde{B}_{i j} g \\
= & \frac{\partial^{2} f(\phi)}{\partial z_{k} \partial z_{l}} \frac{\partial \phi_{k}}{\partial t_{i}} \frac{\partial \phi_{l}}{\partial t_{j}} m+\frac{\partial f(\phi)}{\partial z_{k}}\left(\frac{\partial^{2} \phi_{k}}{\partial t_{i} \partial t_{j}} m+\frac{\partial \phi_{k}}{\partial t_{i}} \frac{\partial m}{\partial t_{j}}+\frac{\partial \phi_{k}}{\partial t_{j}} \frac{\partial m}{\partial t_{i}}\right) \\
& +f(\phi) \frac{\partial^{2} m}{\partial t_{i} \partial t_{j}}+\tilde{A}_{i j}^{k}\left(\frac{\partial f(\phi)}{\partial z_{r}} \frac{\partial \phi_{r}}{\partial t_{k}} m+f(\phi) \frac{\partial m}{\partial t_{k}}\right)+\tilde{B}_{i j} f(\phi) m \\
\equiv & \frac{\partial \phi_{k}}{\partial t_{i}} \frac{\partial \phi_{l}}{\partial t_{j}} m\left(\frac{\partial^{2} f}{\partial z_{k} \partial z_{l}}+A_{k l}^{r} \frac{\partial f}{\partial z_{r}}+B_{k l} f\right)
\end{aligned}
$$

where thus

$$
\begin{aligned}
& \frac{\partial \phi_{k}}{\partial t_{i}} \frac{\partial \phi_{l}}{\partial t_{j}} A_{k l}^{r}=\tilde{A}_{i j}^{k} \frac{\partial \phi_{r}}{\partial t_{k}}+\frac{\partial \phi_{r}}{\partial t_{i} \partial t_{j}}+\frac{\partial \phi_{r}}{\partial t_{i}} \frac{1}{m} \frac{\partial m}{\partial t_{j}}+\frac{\partial \phi_{r}}{\partial t_{j}} \frac{1}{m} \frac{\partial m}{\partial t_{i}} \\
& \frac{\partial \phi_{k}}{\partial t_{i}} \frac{\partial \phi_{l}}{\partial t_{j}} B_{k l}=\tilde{B}_{i j}+\tilde{A}_{i j}^{r} \frac{1}{m} \frac{\partial m}{\partial t_{r}}+\frac{1}{m} \frac{\partial^{2} m}{\partial t_{i} \partial t_{j}}
\end{aligned}
$$

or in more compact notation

$$
\begin{aligned}
& \phi^{*} A=\tilde{A} \cdot d \phi+d^{2} \phi+d \phi \otimes m^{-1} d m+m^{-1} d m \otimes d \phi, \\
& \phi^{*} B=\tilde{B}+\tilde{A} m^{-1} d m+m^{-1} d^{2} m .
\end{aligned}
$$

Comparison with the corresponding formulae in the one dimensional case suggest now that we as a generalization of the Schwarzian take the differential form

$$
J^{1 /(n+1)} \sum_{i, j} \frac{\partial J^{-1 /(n+1)}}{\partial t_{i} \partial t_{j}} d t_{i} d t_{j}
$$

where as before

$$
J=\operatorname{det}\left(\frac{\partial \phi_{k}}{\partial t_{i}}\right)
$$


The main object of the present section has been precisely to arrive at this definition. In particular, we find, given $A=0$, the conditions for reduction to the case $B=0$ in the form of a total p.d.e. involving that form.

\section{§7. Bilinear covariants}

We return to the case of one variable. We require the following classical result due to Gordan [Go].

LEMma. Let $f_{k}(k=1,2)$ transform according to the rule

$$
f_{k}(t) \longmapsto f_{k}(\phi(t))(E(t))^{-\nu_{k}} \quad(k=1,2)
$$

where $\nu_{k} \in \mathbb{Z}(k=1,2)$ and

$$
\phi(t)=\frac{a t+b}{c t+d} \quad(\text { with } a d-b c=1), \quad E(t)=c t+d .
$$

Let $s \geq 0$ be any integer such that $\nu_{k} \neq 0,-1, \cdots,-(s-1)(k=1,2)$. Then

$$
J_{s}(t) \stackrel{\text { def }}{=} \sum_{i=0}^{s}(-1)^{s-i}\left(\begin{array}{l}
s \\
i
\end{array}\right) \frac{f_{1}^{(i)}(t)}{\left(\nu_{1}\right)_{i}} \frac{f_{2}^{(s-i)}(t)}{\left(\nu_{2}\right)_{s-i}}
$$

transforms according to the rule

$$
J_{s}(t) \longmapsto J_{s}(\phi(t))(E(t))^{-\nu},
$$

where $\nu=\nu_{1}+\nu_{2}+2 s$ and, generally speaking, $(x)_{i}=x(x+1) \cdots(x+i-1)$ stands for the Pochammer symbol.

The bilinear covariant $J_{s}$ is classically known as the transvectant (German: "Überschiebung"); it was, nearly one hundred years after the appearance of Gordan's book, "rediscovered" in [JP].

On a Riemann surface $X$ with a projective structure it gives rise to a bilinear map

$$
\kappa^{\nu / 2} \times \kappa^{\nu / 2} \longrightarrow \kappa^{\nu / 2},
$$

where $\kappa$ as before stands for the canonical sheaf.

Proof. For the proof take first $\nu_{1}>0, \nu_{2}>0$. Then we can imitate the first proof of Bol's lemma (Section 1), taking

$$
f_{k}(t)=\frac{1}{\left(t-u_{k}\right)^{\nu_{k}}} \quad(k=1,2)
$$


Everything then blows down to the identity

$$
\frac{\left(u_{1}-u_{2}\right)^{s}}{\left(t-u_{1}\right)^{\nu_{1}+s}\left(t-u_{2}\right)^{\nu_{2}+s}}=\sum_{i=0}^{s}(-1)^{s-i}\left(\begin{array}{c}
s \\
i
\end{array}\right) \frac{1}{\left(t-u_{1}\right)^{\nu_{1}+i}\left(t-u_{2}\right)^{\nu_{2}+s-i}} ;
$$

see $[\mathrm{JP}]$.

To carry over the result to the case when $\nu_{1}, \nu_{2}$ have arbitrary sign we argue as follows. Writing $g_{k}=\left(f_{k} \circ \phi\right) E^{-\nu_{k}}(k=1,2)$ we see (e.g. from the unnamed lemma in Section 1) that the expression

$$
\sum_{i=0}^{s}(-1)^{s-i}\left(\begin{array}{l}
s \\
i
\end{array}\right) \frac{g_{1}^{(i)}(t)}{\left(\nu_{1}\right)_{i}} \frac{g_{2}^{(s-i)}(t)}{\left(\nu_{2}\right)_{s-i}}
$$

a priori comes as a polynomial in $c$ and $E$ (and $E^{-1}$ ), whose coefficients in turn are rational functions in $\nu_{1}$ and $\nu_{2}$. More exactly, only terms $E^{k} c^{j}$ with $k=j-\nu$ appear. By the previous proof we know that they must vanish for $\nu_{1}>0, \nu_{2}>0$, hence identically, except for $j=0$. But this means that (1) must agree with $\left(J_{s} \circ \phi\right) E^{-\nu}$.

Remark. If we instead of group actions consider the corresponding infinitesimal actions of the Lie algebra $s l(2, C)$, we get an analogous result with $\nu_{1}, \nu_{2}$ arbitrary complex numbers (see e.g. [M]).

As an application consider the case

$$
\nu_{1}=-(\mu-1), \quad \nu_{2}=2(\mu-s) \quad(\Rightarrow \nu=\mu+1),
$$

where $0 \leq s \leq \mu-1$. Writing $k=\mu-s$ we get for each form $\Theta$ of degree $k$ a linear map

$$
M_{k}^{\theta}: \kappa^{-(\mu-1) / 2} \longrightarrow \kappa^{(\mu+1) / 2} .
$$

Example. Here are some of the operators of lowest degree (and up to a constant factor):

$$
\begin{aligned}
& M_{\theta}^{\mu} f=\Theta f, \quad M_{\theta}^{\mu-1} f=\Theta f^{\prime}+\frac{1}{2} \Theta^{\prime} f, \\
& M_{\theta}^{\mu-2} f=\Theta f^{\prime \prime}+\Theta^{\prime} f^{\prime}+\frac{\mu-1}{2(2 \mu-3)} \Theta^{\prime \prime} f,
\end{aligned}
$$

Thus if our Riemann surface $X$ comes equipped with a projective structure we can, given forms $\Theta_{1}, \Theta_{2}, \cdots, \Theta_{\mu}$ of degree $1,2, \cdots, \mu$ respectively, form the $\mu$ th order linear differential operator $L: \kappa^{-(\mu-1) / 2} \rightarrow \kappa^{(\mu+1) / 2}$ given by 


$$
L=L_{\mu}+M_{\theta_{1}}^{1}+M_{\theta_{2}}^{2}+\cdots+M_{\theta_{\mu}}^{\mu} \text {. }
$$

Conversely, an arbitrary $\mu$ th order linear differential operator, globally defined over $X$, can be viewed as a map from a suitable sheaf $\xi$ into the tensor product $\kappa^{\mu} \otimes \xi$ and gives in a canonical way rise to a projective structure on $X$ and another such differential operator corresponding to $\xi=\kappa^{-(\mu-1) / 2} \otimes \xi_{0}, \xi_{0}$ a flat sheaf (so that $\kappa^{\mu} \otimes \xi=\kappa^{(\mu+1) / 2} \otimes \xi_{0}$ ), i.e. of the form (2) but with $\Theta_{1}=\Theta_{2}=0$.

In this way we happen to recapture the classical Laguerre-Forsyth invariants of $\mu$ th order linear ordinary differential equations (see e.g. [W] or, for a contemporary study, [T]). A different approach to such invariants, directly based on "Bol's lemma" (Section 1), can be found in [B].

\section{§8. An integral formula}

We apply the previous lemma (Section 7) in the case $\nu_{1}=\nu_{2}=$ $-(\mu-1), s=\mu-1$. Then $\nu=0$ and we have thus an "invariant", not a "covariant". In particular, writing $f_{1}=F$, a form of degree $-(\mu-1) / 2$, and taking $f_{2}=\omega^{\mu-1}$, where $\omega$ transforms with degree $-1 / 2$, we have the invariant expression

$$
\Phi=\sum_{k=0}^{\mu-1}(-1)^{k} \frac{\partial^{k}}{\partial z^{k}}\left(\omega^{\mu-1}\right) F^{(\mu-1-k)} .
$$

We can now get a step further and let $\omega$ be of bidegree $(-1 / 2,-1 / 2)$. Thus we do not assume anymore that we are dealing with holomorphic quantities. Then $\Phi$ has bidegree $(0,(1-\mu) / 2)$. If in addition $\omega$ is positive (and globally defined on our Riemann surface $X$ ), then we can associate with $\omega$ a Hermitean metric $d s=|d z| \mid \omega$.

If $z$ is a projective coordinate, it is clear that

$$
\frac{\partial \Phi}{\partial \boldsymbol{z}}=\omega^{\mu-1} F^{(\mu)}+(-1)^{\mu-1} \frac{\partial^{\mu}}{\partial z^{\mu}}\left(\omega^{\mu-1}\right) F .
$$

Let $g$ be a holomorphic differential form of degree $(\mu+1) / 2$, defined in some open subset $\Omega$ of $X$. We now multiply the identity (2) with the conjugate $\bar{g}$. As $\Phi \bar{g}$ is then a $(0,1)$-form or, rather, the coefficient of such an object (that is, $\Phi \bar{g} d \bar{z}$ has an invariant meaning over $\Omega$ ), we can write the result as

$$
d(\Phi \bar{g} d \bar{z})=L_{\mu} F \bar{g} \omega^{\mu-1} d z d \bar{z}+(-1)^{\mu-1} F \bar{g} L_{\mu} \omega^{\mu-1} d z d \bar{z} .
$$

Integrating this yields 


$$
\int_{\Omega} L_{\mu} F \overline{\mathrm{g}} \omega^{\mu-1} d z d \bar{z}+(-1)^{\mu-1} \int_{\Omega} F \overline{\mathrm{g}} L_{\mu} \omega^{\mu-1} d z d \bar{z}=\int_{\partial \Omega} \Phi \overline{\mathrm{g}} d \bar{z}
$$

Until now $\omega$ has been quite general. It is now natural to put conditions on $\omega$ (and the metric), connecting it with the projective structure and the Bol operator(s) $L_{\mu}$ : Indeed, we shall from now on assume that

$$
\begin{array}{ll}
\text { 1. } L_{\mu} \omega^{\mu-1}=0 & \text { for all } \mu, \\
\text { 2. } \omega=0 & \text { on } \partial \Omega .
\end{array}
$$

Clearly 2. implies that the metric is complete in some sense. On the other hand 1. is equivalent to $L_{2} \omega=0(\mu=2)$. (Indeed, this condition implies that $\omega(z)=a z \bar{z}+b z+\bar{b} \bar{z}+c, a, b, c$ being constants ( $a, c$ real and $b$ complex), in terms of a (any) projective coordinate $z$, and then $L_{\mu} \omega^{\mu-1}=0$ for all $\mu$.) Thus 1. implies that the curvature is constant and also that

$$
q=-\frac{\partial^{2} \omega / \partial z^{2}}{\omega}
$$

where $q$ is the coefficient connected with the projective structure (i.e. $\left.L_{2}=\partial^{2} / \partial z^{2}+q\right)$; here $z$ is any local coordinate, not necessarily one subordinated to the projective structure. See Sections 9, 10 for further discussion.

If 1 . is fulfilled (3) becomes

$$
L_{\mu} F \bar{g} \omega^{\mu-1} d z d \bar{z}=d(\Phi \bar{g} d \bar{z}) .
$$

Thus the left hand side is an exact $(1,1)$-form and the integral can be expressed as a pure boundary integral.

If 2 . is fulfilled then on the boundary $\partial \Omega$ holds

$$
\begin{gathered}
\frac{\partial^{k}}{\partial z^{k}}\left(\omega^{\mu-1}\right)=0, \quad 0 \leq k \leq \mu-2 \\
\frac{\partial^{\mu-1}}{\partial z^{\mu-1}}\left(\omega^{\mu-1}\right)=(\mu-1) !\left(\frac{\partial \omega}{\partial z}\right)^{\mu-1}=(\mu-1) !(-i)^{\mu-1}\left(\frac{d \bar{z}}{d z}\right)^{(\mu-1) / 2}\left|\frac{\partial \omega}{\partial z}\right|^{\mu-1} .
\end{gathered}
$$

Thus (4) in this case becomes

$$
\begin{aligned}
& \int_{\Omega} L_{\mu} F \overline{\mathrm{g}} \omega^{\mu-1} d z d \bar{z}+(-1)^{\mu-1} \int_{\Omega} F \overline{\mathrm{g}} L_{\mu} \omega^{\mu-1} d z d \bar{z} \\
& =(\mu-1) ! i^{\mu-1} \int_{\partial \Omega} F \bar{g}\left|\frac{\partial \omega}{\partial z}\right|^{\mu-1}(d z)^{(1-\mu) / 2}(d \bar{z})^{(1+\mu) / 2} .
\end{aligned}
$$


If both 1. and 2. are fulfilled then $|\partial \omega / \partial z|=$ const on $\partial \Omega$ (if $\omega=a z \bar{z}+$ $b z+\bar{b} \bar{z}+c$ then $|\partial \omega / \partial z|=\sqrt{|b|^{2}-a c}$ on $\{\omega=0\}$ ) so that (5) reduces to

$$
\int_{\Omega} L_{\mu} F \bar{g} \omega^{\mu-1} d z d \bar{z}=\text { const } \int_{\partial \Omega} F \bar{g}(d z)^{(1-\mu) / 2}(d \bar{z})^{(1+\mu) / 2} .
$$

For a different proof of this formula, and an application, see [GP].

\section{§9. Factorization of the Bol operator}

Let $X$ be an arbitrary Riemann surface. A set of functions $\{q(z)\}$ (one $q$ for each coordinate variable $z$ ) is called a projective connection on $X$ if they, under change of coordinates, transform according to

$$
\tilde{q}(\tilde{z})\left(\frac{d \tilde{z}}{d z}\right)^{2}=q(z)-\frac{1}{2}\{\tilde{z}, z\}_{2} .
$$

Similarly, a set of functions $\{r(z)\}$ is called an affine connection on $X$ if they tranform according to

$$
\tilde{r}(\tilde{z}) \frac{d \tilde{z}}{d z}=r(z)-\frac{1}{2}\{\tilde{z}, z\}_{1} .
$$

(The factors $1 / 2$ above are not standard and are introduced here just to conform with our previous notation). Observe that we do not require the functions $q(z)$ or $r(z)$ to be holomorphic in general.

As is implicit in the foregoing, there is a one-to-one correspondence between holomorphic projective connections on $X$ and projective structures on $X$ : if $\{q(z)\}$ is given, the projective coordinates on $\{t\}$ are obtained as solutions of

$$
\frac{1}{2}\{t, z\}_{2}=q(z)
$$

and, conversely, given $\{t\}$ the family $\{q(z)\}$ defined by (1) is a projective connection. In particular, a coordinate $z$ is projective iff in that coordinate $q(z)=0$. The corresponding statements with the word "projective" replaced by "affine" are likewise true. (See e.g. [Gu2], [HS1, 2] for more details. See also [B]).

The following facts are easily seen to be true.

(i) If $f$ is a (not necessarily holomorphic) 1-form or, more generally a form of bidegree $(1, *)$, then

$$
\frac{1}{2} \frac{\partial}{\partial z} \log f
$$


is an affine connection (off the zeros of $f$ ). In particular, if $d s=|d z| \mid \omega(z)$ is a Hermitean metric on $X$ then

$$
-\frac{\partial}{\partial z} \log \omega
$$

is an affine connection.

(ii) If $r$ is an affine connection then $q=\partial r / \partial z-r^{2}$ is a projective connection.

(iii) In the presence of an affine connection $r$ one can define a covariant derivative $\nabla=\nabla_{\alpha}$ mapping $\alpha$-forms into $(\alpha+1)$-forms given by

$$
\nabla_{\alpha} f=\frac{\partial f}{\partial z}-2 \alpha r f
$$

We have now the following generalization of the factorization of the Bol operator $L_{\mu}$ indicated in Section 3 in a special case. It gives, in particular, an alternative way of computing $L_{\mu}$ (cf. Section 2).

TheOREM. Let the Riemann surface $X$ be provided with a projective structure, let $q$ denote the corresponding (holomorphic) projective connection and let $\mu>0$ be an integer. Assuming that $q$ comes from a not necessarily holomorphic, affine connection $r$ as in (ii) above we have, writing $\mu=$ $2 l+1$

$$
L_{\mu}=\nabla_{l} \nabla_{l-1} \cdots \nabla_{-l}
$$

In other words:

$$
\begin{aligned}
L_{\mu} & =\sum_{k=0}^{\mu-1}(D-(\mu-1-2 k) r) \\
& =(D-2 l r)(D-2(l-1) r) \cdots(D+2 l r),
\end{aligned}
$$

with $D=d / d z$.

Proof. By the covariance it is enough to prove (2) in projective coordinates. In a projective coordinate system (2) becomes

$$
D^{2 l+1}=(D-2 l r)(D-2(l-1) r) \cdots(D+2 l r) .
$$

(Here $l \geq 0$ is an integer or a half integer.) Moreover $q=0$, i.e.

$$
D r=r^{2} .
$$

Thus it is enough to prove (3) under the assumption (4). This will be done by induction. 
It is straightforward to check (3) for $l=0$ and $l=1 / 2$. Assume that (3) has been proved for $l-1$, i.e. that

$$
D^{2 l-1}=(D-2(l-1) r) \cdots(D+2(l-1) r) .
$$

Then we get, for $l$ itself

$$
\begin{gathered}
(D-2 l r)(D-2(l-1) r) \cdots(D+2 l r) f=(D-2 l r) D^{2 l-1}(D+2 l r) f \\
=D^{2 l+1} f+2 l D^{2 l}(r f)-2 l r D^{2 l} f-4 l^{2} r D^{2 l-1}(r f) .
\end{gathered}
$$

We have to prove that this equals $D^{2 l+1} f$, i.e. that

$$
D^{2 l}(r f)=r D^{2 l} f+2 l r D^{2 l-1}(r f) .
$$

By (4) $D^{n} r=n ! r^{n+1}$. Thus

$$
\begin{aligned}
D^{2 l}(r f) & =\sum_{k=0}^{2 l}\left(\begin{array}{l}
2 l \\
k
\end{array}\right) D^{k} r D^{2 l-k} f \\
& =r D^{2 l} f+\sum_{k=1}^{2 l} 2 l(2 l-1) \cdots(2 l-k+1) r^{k+1} D^{2 l-k} f \\
& =r D^{2 l} f+2 l r \sum_{k=1}^{2 l}\left(\begin{array}{r}
2 l-1 \\
k-1
\end{array}\right) D^{k-1} r D^{2 l-k} f \\
& =r D^{2 l} f+2 l r D^{2 l-1}(r f)
\end{aligned}
$$

proving (5).

If $X$ is compact of genus one, as in Section 3, then every projective connection comes from an affine connection, as in (ii) above. Hence it is seen that the factorization of $L_{\mu}$ in Section 3 is a special case of the above theorem. However, it is not clear whether, in general, a holomorphic projective connection always comes from an affine connection (not required to be holomorphic). Of course, given the family $\{q(z)\}$ one can always solve $q=\partial r / \partial z-r^{2}$ locally for $r(z)$ (even with $r(z)$ holomorphic) but in general these $r=r(z)$ will not transform as an affine connection. On the other hand (2) may be of interest also locally (i.e. without each factor in (2) having a globally covariant meaning). E.g. if $t$ is a fixed local variable defined on an open subset $V$ of $X$ then the projective connection $\{q(z)\}$ on $V$ given by $q(z)=(1 / 2)\{t, z\}_{2}$ (in terms of arbitrary local variables $\{z\})$ comes from the affine connection $r(z)=(1 / 2)\{t, z\}_{1}$. Thus we have

$$
L_{\mu}=\left(\frac{\partial}{\partial z}-l\{t, z\}_{1}\right)\left(\frac{\partial}{\partial z}-(l-1)\{t, z\}_{1}\right) \cdots\left(\frac{\partial}{\partial z}+l\{t, z\}_{1}\right)
$$


for the Bol operator $L_{\mu}$ belonging to $\{q(z)\}$, i.e. to the projective structure on $V$ for which $t$ is a projective coordinate.

Next, assume that a metric $d s=|d z| / \omega(z)$ is given on $X$. By (i) and (ii) we then get affine and projective connections on $X$ by

$$
r=-\frac{\partial}{\partial z} \log \omega, \quad q=\frac{\partial r}{\partial z}-r^{2}=-\frac{\partial^{2} \omega / \partial z^{2}}{\omega} .
$$

The curvature of $d s$ is given by

$$
K=4 \omega^{2} \frac{\partial^{2} \log \omega}{\partial z \partial \bar{z}}=-4 \omega^{2} \frac{\partial r}{\partial \bar{z}} .
$$

and derivation of this gives

$$
\frac{\partial K}{\partial z}=-4 \omega^{2} \frac{\partial q}{\partial \bar{z}}
$$

Thus we see that $r(z)$ is holomorphic iff the curvature is identically zero and that $q(z)$ is holomorphic iff the curvature is constant.

It is well-known that on any Riemann surface there is, up to a constant factor, exactly one metric $d s$ which is both complete and constant curvature. This is the metric which, via the uniformization theorem, corresponds to one of the metrics with $\omega(t)=1+|t|^{2}, 1$ or $\Im t$ on $\hat{C}=$ $C \cup\{\infty\}, C$ or $\{t \in C: \mathfrak{s} t>0\}$ respectively.

If $X$ is a compact, any metric is automatically complete. Thus we conclude that if $X$ is compact then a holomorphic connection on $X$ comes from a metric only in the above "canonical" case (i.e. with the Poincaré metric in most cases). The corresponding projective structure then of course is the one given by the uniformization theorem.

\section{§10. Remarks on "non-Euclidean geometry"}

In this section, which is largely independent of the rest of the paper but inspired of it, we make some simple observations about Hermitean metrics with constant curvature on a Riemann surface.

Let $X$ be a Riemann surface equipped with a projective structure. Then we can define the operator $\left(L_{2}=\right) L=D^{2}+q$ (where $D=\partial / \partial z$ is derivation with respect to local coordinate $z$ ) which sends $(-1 / 2, *)$ forms into $(3 / 2, *)$-forms. The conjugate operator $\bar{L}=\bar{D}^{2}+\bar{q}$ (where analogously $\bar{D}=\partial / \partial \bar{z})$ sends $(*,-1 / 2)$-forms into $(*,-3 / 2)$-forms. We 
can now define a non-Euclidean geometry as an Hermitean metric $d s=$ $|d z| / \omega(z)$ such that $L \omega=0=\bar{L} \omega$. It is now clear that if $t$ is a projective coordinate $(q(t)=0)$ then $\omega(t)=a t \bar{t}+b t+\bar{b} \bar{t}+c$ (a,c real, $b$ complex). That is, the "absolute" (that is, the set where the metric degenerates) is a "circle" (possibly imaginary-elliptic geometry, or degenerate-parabolic geometry).

We can also fit curvature into this picture. Define the latter as usual (see Section 9) via

$$
K=4 \omega^{2} \bar{D} D \log \omega
$$

Formula:

$$
d K=4 \omega^{2} \bar{D}\left(\frac{L \omega}{\omega}\right) d z+4 \omega^{2} D\left(\frac{\bar{L} \omega}{\omega}\right) d \bar{z}
$$

Proof. We may work in a projective coordinate $(q=0)$. Then

$$
\omega^{2} \bar{D} \frac{L \omega}{\omega}=\omega^{2} \bar{D}\left(\frac{D^{2} \omega}{\omega}\right)=\omega^{2}\left(\frac{\bar{D} D^{2} \omega}{\omega}-\frac{\bar{D} \omega \cdot D^{2} \omega}{\omega^{2}}\right) .
$$

On the other hand

$$
\begin{aligned}
\frac{1}{4} \cdot D K= & \omega^{2} \bar{D} D^{2} \log \omega+D \omega^{2} \cdot \bar{D} D \log \omega \\
= & \omega^{2} \bar{D}\left(\frac{D^{2} \omega}{\omega}-\frac{(D \omega)^{2}}{\omega^{2}}\right)+2 \omega D \omega \cdot \bar{D}\left(\frac{D \omega}{\omega}\right) \\
= & \omega^{2}\left(\frac{\bar{D} D^{2} \omega}{\omega}-\frac{\bar{D} \omega \cdot D^{2} \omega}{\omega^{2}}-2 \frac{D \omega \cdot \bar{D} D \omega}{\omega^{2}}+2 \frac{(D \omega)^{2} \bar{D} \omega}{\omega^{3}}\right) \\
& +2 \omega D \omega\left(\frac{\bar{D} D \omega}{\omega}-\frac{D \omega \cdot \bar{D} \omega}{\omega^{2}}\right) \\
= & \omega^{2}\left(\frac{\bar{D} D^{2} \omega}{\omega}-\frac{\bar{D} \omega \cdot D^{2} \omega}{\omega^{2}}\right) \cdot
\end{aligned}
$$

As $d=D d z+\bar{D} d \bar{z}$ this completes the proof.

We see thus that $K=$ const in non-Euclidean geometry.

\section{REFERENCES}

[B] G. Bol, Invarianten linearer Differentialgleichungen, Abh. Math. Sem. Univ. Hamburger Univ., 16 (1949), 1-28.

[E] M. Eichler, Eine Verallgemeinerung der Abelschen Integrale, Math. Z., 67 (1957), 267-298.

[Go] P. Gordan, Invariantentheorie, Teubner, Leipzig, 1887. 
[Gu1] R. G. Gunning, Special coordinate coverings of Riemann surfaces, Math. Ann., 170 (1967), 67-86.

[Gu2] - Lectures on Riemann surfaces, Princeton University Press, Princeton, 1966.

[Gu3] - On uniformization of complex manifolds: the role of connections, Princeton University Press, Princeton, 1980.

[GP] B. Gustafsson - J. Peetre, Hankel forms on multiply connected plane domains, Part two, The case of higher connectivity, Complex Variables (to appear).

[HS1] N. S. Hawley - M. Schiffer, Half-order differentials on Riemann surfaces, Acta Math., 115 (1966), 199-236.

[HS2] - Connections and conformal mappings, Acta Math., 107 (1962), 175-274.

[JP] S. Janson-J. Peetre, A new generalization of Hankel operators (the case of higher weights), Math. Nachr., 132 (1987), 313-328.

[K] I. Kra, Automorphic forms and Kleinian groups, Benjamin, Reading, 1972.

[M] H. Morikawa, Some analytic and geometric applications of the invariant theoretic methods, Nagoya Math. J., 80 (1980), 1-47.

[N] N. Nörlund, Vorlesungen über Differenzenrechnung, Springer, Berlin, 1924.

[P] J. Peetre, Invariant function spaces connected with the holomorphic discrete series, in: P. L. Butzer (ed.), Anniversary volume on approximation and functional analysis, pp.119-134, Birkhäuser, Basel-Boston-Stuttgart, 1984.

[R] W. Rudin, Function theory on the unit ball of $C^{n}$, Springer, New York-HeidelbergBerlin, 1980.

[T] Y. Teranishi, The variational theory of higher-order linear differential equations, Nagoya Math. J., 95 (1984), 137-161.

[W] E. J. Wilczynski, Projective differential geometry of curves and ruled surfaces, Chelsea, New York, 1961.

Björn Gustafsson

Matematiska institutionen

$K T H$

S-100 44 STOCKHOLM

Sweden

Jaak Peetre

Matematiska institutionen

Stockholms universitet

Box 6701

S-113 85 Stockholm

Sweden 